\title{
Desenvolvimento de aplicativos móveis com AppInventor por alunos do ensino médio: relato de um projeto de extensão com foco em acessibilidade
}

\author{
Herison Maciel, Marília Mendes e Anna Beatriz Marques \\ Universidade Federal do Ceará (UFC) - Russas, CE - Brasil \\ herisonmaciel@alu.ufc.br, \{marilia.mendes, beatriz.marques\}@ufc.br
}

Abstract. The integration of information technology in schools enables the development of computational thinking and the implementation of ideas that solve society's problems. The extension projects are opportunities to integrate academic research to the needs of a community. This paper reports an extension project that aimed to develop accessible technologies for people with Autistic Spectrum Disorder (ASD) of a country town. In order to develop the computational thinking and awareness about the importance of accessibility, the project offered to high school students a course of developing applications with App Inventor, during which topics about accessibility were discussed. As a result, students have developed four applications and they indicated a greater interest in computer science.

Resumo. A inserção da informática nas escolas possibilita o desenvolvimento do pensamento computacional e a implementação de ideias que solucionem problemas da sociedade. Os projetos de extensão são oportunidades de integrar pesquisas acadêmicas às necessidades de uma comunidade. Este artigo relata um projeto de extensão que visa o desenvolvimento de tecnologias acessíveis para pessoas com Transtorno do Espectro Autista (TEA) de um município do interior. Com o intuito de desenvolver o pensamento computacional e a conscientização sobre a importância da acessibilidade, o projeto ofertou para alunos do ensino médio um curso de desenvolvimento de aplicativos com AppInventor durante o qual foram abordados temas relacionados à acessibilidade. Como resultado, os alunos desenvolveram quatro aplicativos e indicaram um maior interesse pela área de Computação.

\section{Introdução}

A inserção do ensino do pensamento computacional e práticas de programação no contexto escolar trazem benefícios para o desenvolvimento do aluno. Conforme relata SCAICO et al. (2013), "este tipo de educação permite o desenvolvimento de diversas capacidades que contribuem para melhorar o raciocínio lógico dos estudantes”. O ensino de programação nas escolas pode contribuir para apresentar aos estudantes novas possibilidades de uso das tecnologias que habitualmente manuseiam, além de apoiar o desenvolvimento de habilidades de abstração, raciocínio lógico, decomposição e resolução de problemas [FINIZOLA et al. 2014]. 
VII Congresso Brasileiro de Informática na Educação (CBIE 2018)

Anais do XXIV Workshop de Informática na Escola (WIE 2018)

A prática inicial de programação deve ser suportada por ferramentas computacionais, que permitam ao aluno experimentar, descobrir, testar e errar suas respostas [DELGADO et al. 2004]. Estas propostas apresentam situações de aprendizagem que envolvem diversos recursos, abrangendo a utilização de linguagens de programação visual [FRANÇA et al. 2012; SCAICO et al. 2012]. Bordin et al. (2017) indicam a necessidade da condução de projetos que objetivem estimular o pensamento computacional através da programação em escolas públicas.

Neste contexto, um projeto de extensão foi iniciado com o intuito de desenvolver habilidades de raciocínio lógico e desenvolvimento de sistemas acessíveis aos estudantes do ensino médio da cidade de Russas/CE. A finalidade do projeto consistia no desenvolvimento de aplicativos acessíveis voltados para resolução de problemas de pessoas com deficiência. Segundo o Art. $1^{\circ}$ da Convenção sobre os Direitos das Pessoas com Deficiência, pessoas portadoras de deficiência são aquelas que possuem determinadas restrições de longa duração, podendo ser essas restrições de caráter mental, intelectual, física ou sensorial e que, com a interação com o ambiente, denigra a participação total e de maneira efetiva com a sociedade em igualdades de condições com o resto da população [BRASIL, 2009].

Este projeto também visa estimular o interesse dos alunos do município para os cursos de Ciência da Computação e Engenharia de Software, ofertados no campus de uma universidade situada no município. Para alcançar os objetivos mencionados, foi conduzido um curso de desenvolvimento de aplicativos com a ferramenta App Inventor para alunos do ensino médio do município. O curso foi conduzido ao longo de sete meses e como resultado, os alunos desenvolveram quatro diferentes aplicativos móveis para o sistema operacional Android. Neste artigo, a experiência com o projeto de extensão e os resultados alcançados são apresentados.

Este artigo está organizado da seguinte forma: na Seção 2 é descrita a ferramenta que foi utilizada para o desenvolvimento das aplicações. A seção 3 apresenta os trabalhos relacionados. A Seção 4 apresenta a metodologia utilizada no projeto de extensão, seu planejamento e desenvolvimento. A Seção 5 apresenta os resultados alcançados. Por fim, a Seção 6 indica as considerações finais e lições aprendidas.

\section{Ferramenta utilizada para a criação de aplicativos - App Inventor}

O App Inventor Team foi liderado por Hal Abelson e uma equipe do Google Education, atualmente mantido pelo MIT (Massachusetts Institute of Technology). Essa plataforma permite criar programas para o sistema operacional Android por meio da programação baseada em blocos, no estilo quebra cabeça com o mouse. O App Inventor permite que usuários, mesmo que iniciantes em programação ou usuários comuns, possam desenvolver aplicativos totalmente funcionais para a plataforma de dispositivos móveis Android [MIT, 2012].

A plataforma é online e disponibiliza a funcionalidade de teste em tempo real através de Cabo USB, Wifi ou emulador no próprio Computador (PC). Por conta de sua programação facilitada, sua interface simples e um visual elegante, o App Inventor é utilizado para o ensino da lógica de programação. A Figura 1 ilustra a interface web da ferramenta App Inventor. 
VII Congresso Brasileiro de Informática na Educação (CBIE 2018)

Anais do XXIV Workshop de Informática na Escola (WIE 2018)

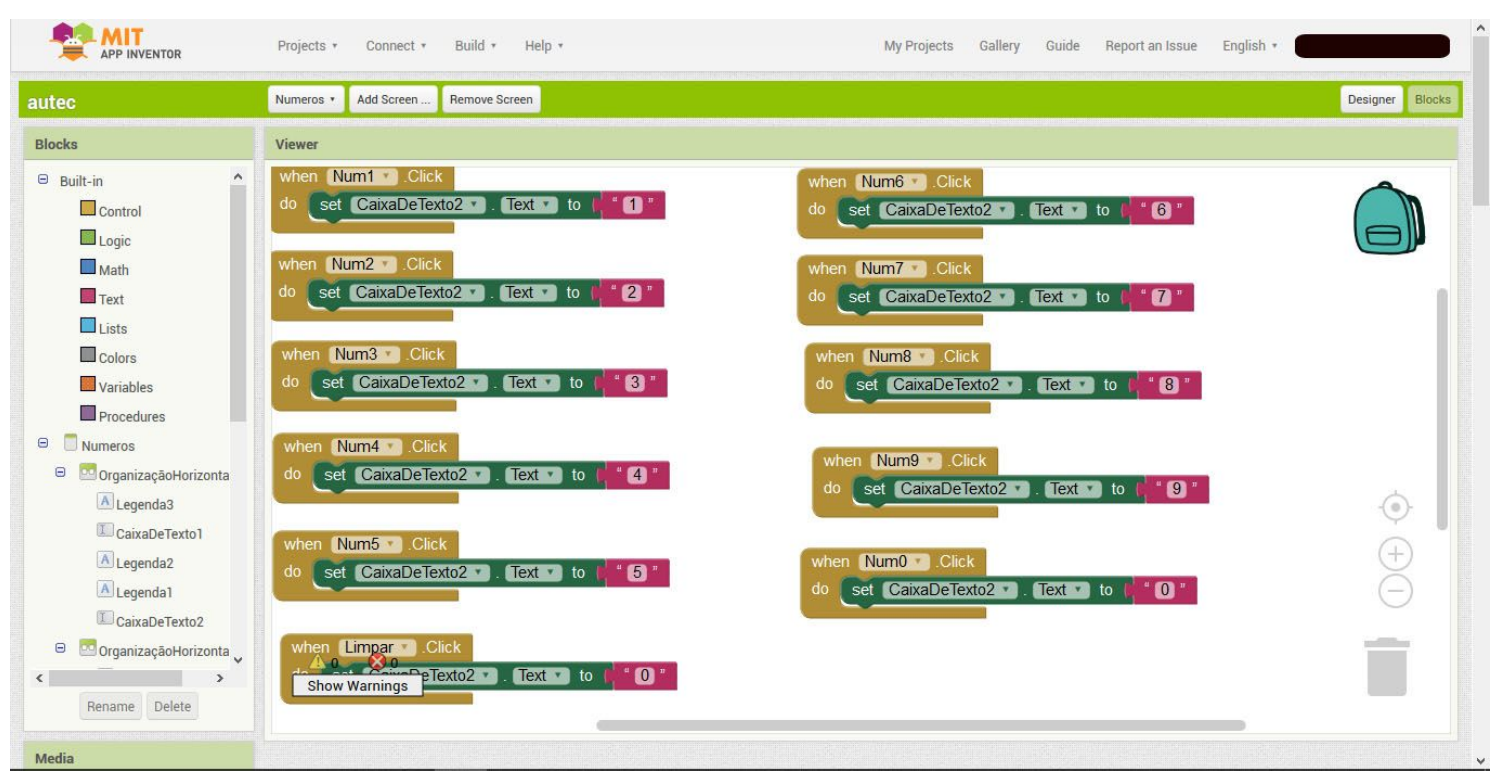

Figura 1. Interface web do App Inventor.

É necessário buscar formas diversificadas de utilizar recursos motivacionais e analogias que despertem o interesse dos alunos e facilite o aprendizado dos conceitos de programação [FINIZOLA et al. 2014]. Neste sentido, essa ferramenta pode ser utilizada por pessoas iniciantes que gostem de programação pois não é tão complicada e possibilita a criação de suas primeiras aplicações.

\section{Trabalhos Relacionados}

Bordin et al. (2017) relatam uma ação extensionista que promoveu uma oficina de programação com o App Inventor por meio de dois encontros, totalizando seis horas de oficina. Os resultados indicaram o interesse dos alunos pela área de Computação e pela participação em outras atividades promovidas pela ação de extensão. Ribeiro et al. (2016) propõe dinâmicas para adoção do App Inventor para o desenvolvimento de aplicativos móveis com alunos de ensino médio. Os autores concluem que o App Inventor oferece oportunidades para o aprendizado e fornece aos participantes uma nova visão sobre programação e sobre a carreira na área de computação.

Gomes et al. (2013) apresentam um panorama sobre o potencial didático-pedagógico do App Inventor além de relatarem experiências com o uso da ferramenta para o ensino de lógica de programação a alunos do ensino médio e ensino superior por meio de oficinas. Finizola et al. (2014) apresentam um relato de experiência em um curso de ensino de programação utilizando o App inventor com alunos do ensino médio. Os resultados apontam que o aprendizado de programação é influenciado pelo uso de estratégias que envolvam a resolução de problemas, uso de analogias com o cotidiano e que promovam desafios individuais.

O diferencial da pesquisa relatada neste artigo é que o App Inventor foi adotado em um curso de longa duração com o intuito de desenvolver aplicativos acessíveis. Assim, além das habilidades de programação, a importância da acessibilidade em tecnologias de software também foi abordada. 
VII Congresso Brasileiro de Informática na Educação (CBIE 2018)

Anais do XXIV Workshop de Informática na Escola (WIE 2018)

\section{Projeto de Extensão: planejamento e desenvolvimento}

O projeto de extensão foi iniciativa de uma professora especialista em Interação Humano-Computador (IHC) em 2017. A equipe inicial do projeto era formada pela coordenadora, um aluno bolsista de extensão e um aluno bolsista de iniciação acadêmica. Durante o planejamento do projeto, os envolvidos decidiram oferecer um curso de desenvolvimento de aplicativos móveis com foco em acessibilidade para os alunos do ensino médio do município. A coordenadora do projeto e os alunos bolsistas envolvidos elaboraram o plano de aulas do curso, conforme ilustra a Tabela 1.

Os conteúdos abordados seriam relacionados à programação [PRO] e técnicas de design centrado no usuário [IHC]. O curso foi organizado em duas fases: na Fase 1, o objetivo consistiu em abordar os conteúdos necessários para o desenvolvimento dos aplicativos acessíveis; na Fase 2, o foco foi no desenvolvimento dos aplicativos por equipe de alunos.

Tabela 1. Resumo do conteúdo abordado no curso.

\begin{tabular}{|l|l|}
\hline Etapa & Conteúdo abordado \\
\hline $\begin{array}{l}\text { Fase 1 } \\
\text { aulas })\end{array}$ & $\begin{array}{l}\text { [PRO] História da Computação e componentes dos computadores. O que é um } \\
\text { software? Tipos diferentes de software e sua evolução. Introdução à Lógica de } \\
\text { Programação com jogos da plataforma Code.Org. Conceitos básicos de } \\
\text { programação. Comandos de decisão. Comando de repetição. Uso de vetores. Uso } \\
\text { de funçães. Programação com a ferramenta App Inventor. } \\
{[\text { IHC] Processo de desenvolvimento de software. Por que trabalhar com }} \\
\text { acessibilidade? Usabilidade Experiência do usuário e Acessibilidade. } \\
\text { Modelagem de usuários por meio de personas. Modelagem do contexto de uso. } \\
\text { Prototipação de baixa fidelidade e alta fidelidade. Testes com usuários. }\end{array}$ \\
\hline $\begin{array}{l}\text { Fase 2 } \\
\text { aulas })\end{array}$ & $\begin{array}{l}\text { Organização dos times para os projetos de desenvolvimento dos aplicativos. } \\
\text { Apresentação dos resultados das visitas realizadas pelos alunos bolsistas do } \\
\text { projeto de extensão. Elaboração das personas e contexto de uso do aplicativo a } \\
\text { partir dos dados coletados nas visitas aos colégios. Prototipação de baixa } \\
\text { fidelidade dos aplicativos a serem desenvolvidos. Avaliação da qualidade dos } \\
\text { protótipos. Desenvolvimento dos aplicativos. }\end{array}$ \\
\hline
\end{tabular}

$\mathrm{Na}$ etapa de preparação do curso, os alunos bolsistas fizeram visitas em três escolas públicas do ensino médio do município com o intuito de divulgar o curso e o período de inscrições. As inscrições foram realizadas por meio de um formulário do GoogleForms $^{1}$, de maneira online. No total, foram recebidas 200 inscrições. Inicialmente, a coordenadora havia definido que seriam ofertadas 20 vagas. Devido à demanda, decidiu-se ofertar 32 vagas. Os alunos foram selecionados considerando o colégio no qual estavam matriculados e os horários disponíveis, assim foi balanceado os alunos por escolas.

Após a seleção dos alunos que iriam participar do curso, as aulas foram iniciadas. As aulas eram realizadas duas vezes por semana com duração de 1 hora e 30 minutos, no período da noite. Na Fase 1, os dois alunos bolsistas atuaram como

\footnotetext{
${ }^{1}$ https://www.google.com/intl/pt-BR/forms/about/
} 
VII Congresso Brasileiro de Informática na Educação (CBIE 2018)

Anais do XXIV Workshop de Informática na Escola (WIE 2018)

instrutores, abordando os conteúdos sobre planejamento, prototipação e desenvolvimento de aplicativos. Os alunos bolsistas prepararam seu próprio material didático, com a supervisão da coordenadora e utilizando a metodologia empregada na maratona de aplicativos [MARATONA APP, 2014].

As aulas foram realizadas em salas de aulas e laboratórios de informática nos quais era disponibilizado um computador por aluno. Nas salas de aula, os alunos bolsistas repassaram o conteúdo com slides e dinâmicas com o intuito de melhorar o aprendizado dos alunos e a interação social da turma. As aulas realizadas nos laboratórios tiveram como objetivo abordar conteúdos sobre IHC, pesquisa de aplicativos existentes voltados para área de acessibilidade, atividades de lógica de programação com Code.org e o desenvolvimento de aplicativos com AppInventor.

Durante a Fase 1, outros três alunos da universidade começaram a fazer parte da equipe do projeto como voluntários. A fim de definir o público alvo dos aplicativos, foi realizada uma investigação pelos alunos bolsistas e voluntários do projeto na secretaria de educação do município. Durante a pesquisa, foi relatado que haviam 50 alunos com Transtorno do Espectro do Autismo (TEA) no município, de acordo com censo de 2017. A maioria dos alunos com TEA eram crianças e adolescentes entre 5 e 15 anos, matriculados no ensino infantil e séries iniciais do ensino fundamental. A partir dessa pesquisa, foi definido que o foco do projeto seria o autismo por ser a maior demanda de pessoas com deficiência no município.

Estudos relatam que crianças com TEA demonstram interesse em manusear e utilizar recursos computacionais como celular, tablet e jogos eletrônicos [Avila et al. 2013]. Por conta disso, esses recursos são inseridos cada vez mais nas terapias e processos de ensino e aprendizagem dessas crianças [Passerino et. al. 2006]. Ainda por meio de pesquisas realizadas pela equipe do projeto de extensão, observou-se que a prefeitura do município disponibiliza uma pessoa denominada de "cuidador" para acompanhar esses alunos durante suas aulas. Contudo, esses cuidadores em sua maioria não possuem formação na área de autismo, mas realizavam debates sobre o tema e compartilham experiências em reuniões mensais com todos os cuidadores do município.

A Fase 2 do curso foi direcionada ao desenvolvimento dos aplicativos. Os alunos foram organizados em quatro equipes para o desenvolvimento de aplicativos destinados a pessoas com TEA. Cada equipe escolheu o seu próprio aplicativo depois de algumas realizações de reuniões e debates entre os próprios membros da equipe, alunos bolsistas e com a coordenadora do projeto. Com isso, iniciou-se o desenvolvimento dos aplicativos móveis utilizando a ferramenta App Inventor.

Os alunos planejaram como os aplicativos seriam desenvolvidos, realizando pesquisas, recebendo informações sobre as pesquisas conduzidas no município e aplicando as técnicas de prototipação de baixa e de alta fidelidade. Em seguida, os alunos desenvolveram os aplicativos. Na Fase 2, os alunos bolsistas atuavam como gerentes de projeto, sendo que cada um ficou responsável por gerenciar as atividades de duas equipes. Os aplicativos foram projetados com o acompanhamento dos alunos bolsistas e voluntários do projeto, que realizaram pesquisas sobre o desenvolvimento de aplicativos para pessoas com TEA e com isso, puderam validar os protótipos ao longo do desenvolvimento. As equipes de alunos desenvolveram quatro aplicativos móveis com o intuito de prover benefícios, tanto para as pessoas com TEA, como para os 
VII Congresso Brasileiro de Informática na Educação (CBIE 2018)

Anais do XXIV Workshop de Informática na Escola (WIE 2018)

cuidadores. Os aplicativos foram denominados pelas equipes de AutPlus, Autec, Guia do Cuidador e AutQuiz que serão apresentados na próxima seção.

\section{Resultados Alcançados}

Os resultados alcançados serão descritos nos seguintes tópicos: aplicações desenvolvidas e percepção dos alunos de ensino médio.

\subsection{Aplicativos desenvolvidos}

Em relação aos quatro aplicativos desenvolvidos, o AutPlus (Figura 2) resumidamente é um aplicativo que serve principalmente para o desenvolvimento da interação do autista com a sociedade, contendo recursos para se comunicarem e expressarem suas necessidades. Os usuários poderão se comunicar expressando seus sentimentos, dores físicas e ações desejadas selecionando uma imagem (botão). Em seguida, o aplicativo emite o som associado à imagem.
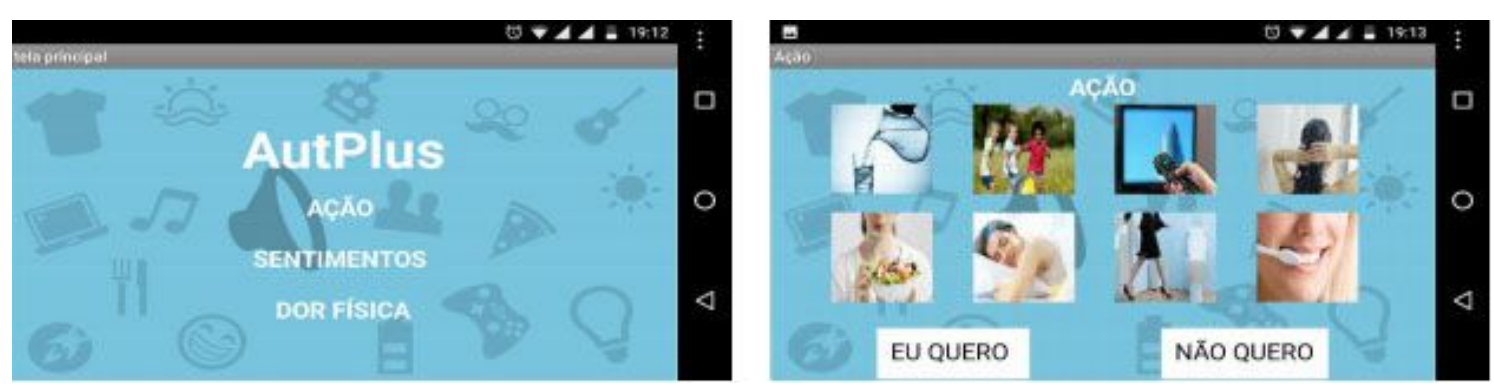

Figura 2. Tela inicial do aplicativo AutPlus e tela de ações do aplicativo AutPlus

O aplicativo Autec (Figura 3) é um aplicativo educacional como objetivo de ensinar por meio de jogos educativos com números, letras, cores e palavras. $\mathrm{O}$ aplicativo é indicado para crianças de 5 a 8 anos.

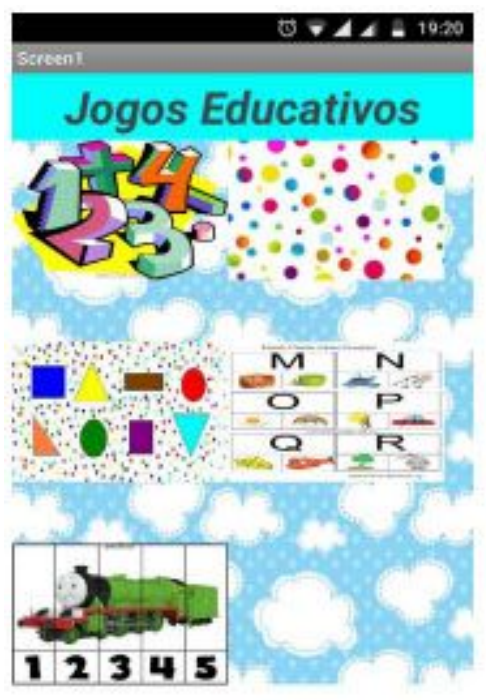

$\triangleleft$

○

$\square$

$\triangleleft$

$\circ$

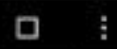

Figura 3. Tela inicial do aplicativo Autec e um jogo de montagem de palavras 
VII Congresso Brasileiro de Informática na Educação (CBIE 2018)

Anais do XXIV Workshop de Informática na Escola (WIE 2018)

O Guia do Cuidador (Figura 4) visa auxiliar o cuidador do autista em situações do dia a dia e disponibilizar informações sobre o TEA, disponibilizando curiosidades e brincadeiras direcionadas para a interação com uma pessoa autista.
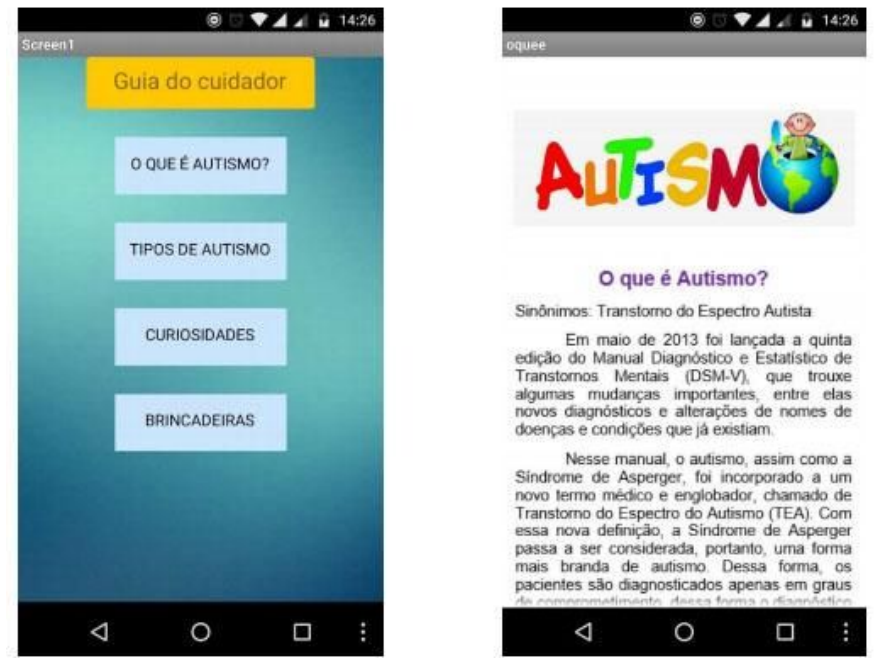

Figura 4. Tela inicial e tela o que é Autismo? Do aplicativo Guia do Cuidador

Por fim, o aplicativo AutQuiz (Figura 5) tem o intuito de auxiliar na memorização de conteúdos escolares, uma vez que o TEA pode desencadear dificuldades de aprendizado. Então, o AutQuiz disponibiliza jogos de perguntas e respostas relacionados ao conteúdos de Matemática, Geografia, Português e História para apoiar o aprendizado e a memorização dos conteúdos.
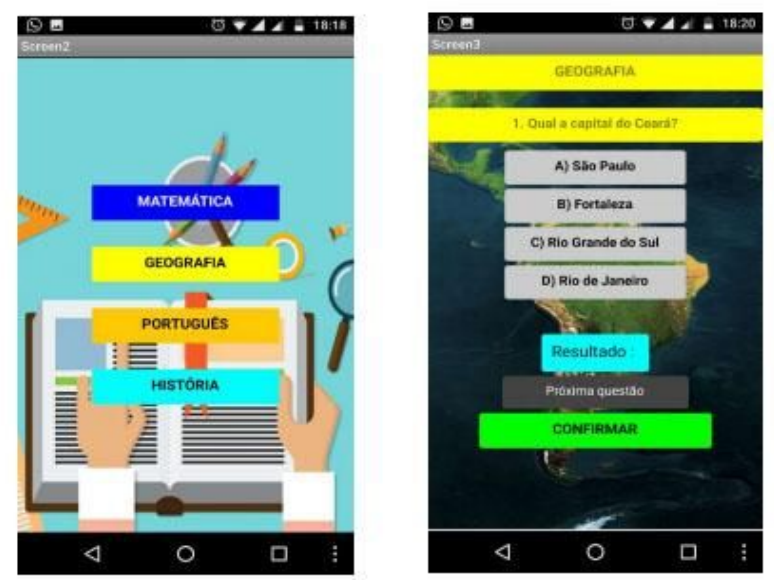

Figura 5. Tela menu principal e pergunta do tema de geografia do aplicativo AutQuiz

\subsection{Percepção dos alunos do ensino médio que finalizaram o curso}

Durante o curso, observou-se uma evasão de alunos, principalmente na segunda fase do projeto. Alguns alunos iniciaram outros projetos, outros alunos disseram que não estavam conseguindo conciliar o tempo com o projeto, pois uma das escolas era de tempo integral e os alunos estavam se sentindo muito cansados. Por conta da distância 
VII Congresso Brasileiro de Informática na Educação (CBIE 2018)

Anais do XXIV Workshop de Informática na Escola (WIE 2018)

de suas residências, também teve desistência por motivo de deslocamento e pelo fato de não conseguirem entender a lógica de programação na primeira fase.

Ao término do ano de 2017, ao todo, 17 alunos concluíram o curso. Na última aula, 14 desses alunos responderam a um questionário. De maneira geral, observou-se que os alunos ficaram satisfeitos com os seus próprios resultados e com um desejo maior de aprendizagem. O Gráfico 1 ilustra as respostas dos alunos às questões objetivas do questionário.

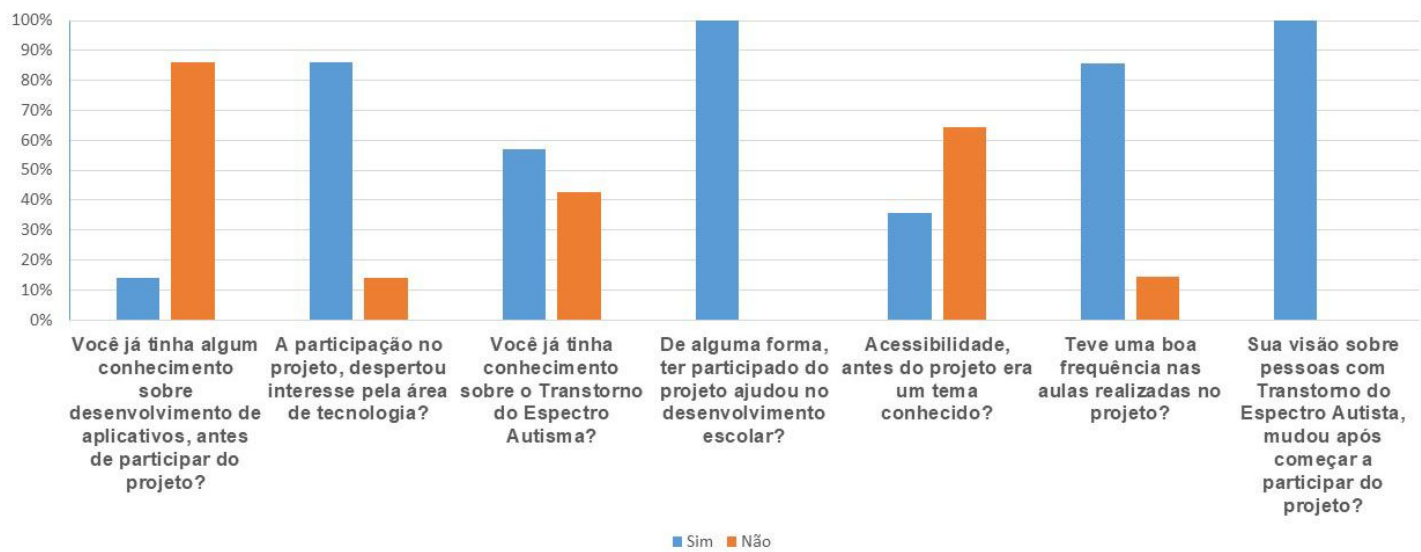

Gráfico 1. Perguntas feitas aos alunos ao término do curso.

Mais de $50 \%$ dos alunos concluintes indicaram que antes do curso, não possuíam conhecimento sobre o desenvolvimento de aplicativos, a acessibilidade e o Transtorno do Espectro Autista. Isso indica que o curso ofereceu a oportunidade de ampliar conhecimentos em áreas até então pouco conhecidas e exploradas pelos alunos.

Acima de $80 \%$ dos alunos indicaram que a participação no projeto despertou o seu interesse pela área de tecnologia. Um dos alunos concluintes ingressou no semestre seguindo no curso de Engenharia de Software na mesma universidade na qual foi oferecido o curso de extensão. Todos os alunos concluintes indicaram que a participação no projeto ajudou no desenvolvimento escolar. Mais de $80 \%$ dos alunos também indicaram que tiveram uma boa frequência nas aulas realizadas no projeto. Por fim, todos os alunos indicaram que a sua visão sobre pessoas com Transtorno do Espectro Autista, mudou após começar a participar do projeto. Nas perguntas abertas mas não obrigatórias tivemos os seguintes resultados:

(1) Você teria interesse em cursar algum curso da UFC? Se sim, Qual? - 71,43 $\%$ das respostas dessa pergunta foi "sim", e o curso com maior citação foi o de Engenharia Civil, seguido por Engenharia de Software e Ciência da Computação.

(2) Depois de ter trabalhado com o desenvolvimento de aplicativos para autistas, o que você considera como acessibilidade? - Foi possível notar com base nas respostas, que os estudantes consideram a acessibilidade como um meio de ajudar o portador da deficiência na sua inclusão, fazendo com que eles tenham as mesmas oportunidades de aprendizado e comunicação que qualquer outra pessoa, através dos aplicativos. Para exemplificar, destacamos as seguintes respostas:

Resposta 1 - "Oportunidade para todos, independente se você é portador de alguma deficiência física ou mental”. 
VII Congresso Brasileiro de Informática na Educação (CBIE 2018)

Anais do XXIV Workshop de Informática na Escola (WIE 2018)

Resposta 2 - "Uma forma de ajudar as pessoas que precisam a ter acesso às mesmas oportunidades".

(3) O que lhe despertou interesse para participar do projeto? - Analisando as respostas, notou-se que os alunos desejavam ampliar seus conhecimento e o fato de o projeto ser da área da tecnologia também despertou o interesse dos alunos.

(4) Sugestões e/ou críticas? - Todos sugeriram que o campus ofertasse mais projetos com a participação da comunidade e que a duração do projeto seja maior.

(5) Caso haja continuidade do projeto de extensão, você teria interesse em continuar participando? - Para essa pergunta verificou-se que a $85,71 \%$ dos alunos queriam continuar e ainda sugeriram o aumento de horas do curso.

Com base nos resultados, é possível considerar que os objetivos do projeto em (i) conscientizar os alunos sobre a importância da acessibilidade; (ii) desenvolver habilidades de desenvolvimento de aplicações para resolução de problemas do município foi alcançado; (iii) despertar o interesse dos alunos para os cursos de graduação ofertados pelo campus. A percepção dos alunos fornecidas pelos questionários e os aplicativos desenvolvidos permitem tal conclusão.

\section{Considerações Finais e Lições Aprendidas}

Com esse projeto de extensão, foi possível observar que existe a necessidade de ações que estimulem o pensamento computacional para a resolução de problemas reais de uma comunidade. Contudo, projetos dessa natureza envolvem muitos recursos e tempo de trabalho, recursos materiais (computadores, smartphones, salas, laboratórios, etc.) e humanos (discentes envolvidos e docentes) para planejamento e realização das atividades. Notou-se que o projeto foi bem aceito desde o começo, as pessoas responsáveis pelas escolas envolvidas apoiaram a divulgação do curso. Os alunos demonstraram muito interesse em participar do projeto. E, apesar, da evasão de alguns alunos, os alunos concluintes desenvolveram aplicativos direcionados a pessoas com TEA e forneceram percepções positivas sobre sua experiência durante o curso.

Com a renovação do projeto no ano de 2018, os aplicativos desenvolvidos estão sendo avaliados por meio de testes com usuários e inspeções com base em diretrizes para interfaces direcionadas a pessoas com TEA. O plano de aulas do curso foi repensado e organizado em módulos: 1- História da computação 2- Experiência dos usuários com usos de aplicativos, 3- Lógica de programação, 4- Desenvolvimento mobile com android (parte 1), 5- Transtorno do espectro do autismo (TEA), 6- Processo de desenvolvimento de aplicativos 7- Projeto de interface para usuários com TEA, 8Desenvolvimento ágil, 9- Desenvolvimento mobile com android (parte 2), 10Avaliação e melhoria de interface para usuários com TEA. A equipe do projeto agora é composta por quatro alunos bolsistas, quatro alunos voluntários e duas professoras especialistas em IHC. Um novo curso foi iniciado para o desenvolvimento de novos aplicativos. Após a divulgação do curso nas escolas, foram selecionados 32 alunos de ensino médio. Em paralelo ao curso, estão sendo conduzidas pesquisas com pais,mães e/ou responsáveis por pessoas com TEA e profissionais especializados em TEA, para investigar o uso das tecnologias de software no cotidiano e compreender as necessidades e problemas enfrentados. 
VII Congresso Brasileiro de Informática na Educação (CBIE 2018)

Anais do XXIV Workshop de Informática na Escola (WIE 2018)

\section{Referências}

Avila, B. G., Passerino, L. M., \& Tarouco, L. M. R. (2013). Usabilidade em tecnologia assistiva: estudo de caso num sistema de comunicação alternativa para crianças com autismo. RELATEC: Revista Latinoamericana de Tecnología Educativa, 12(2), 115-129.

Bordin, A., Pinheiro, C. G., Gonçalves, N. N. V., Mombach, J., \& Souza, P. S. (2017). Ensino de programação para o ensino médio com App Inventor: Um relato de experiência extensionista através da colaboração interinstitucional. In Anais do Workshop de Informática na Escola (Vol. 23, No. 1, p. 1179).

BRASIL. Decreto 6.949, de 25 de Agosto de 2009. Convenção sobre os Direitos das Pessoas com Deficiência e seu Protocolo Facultativo. Disponível em: <http://www.planalto.gov.br/ccivil_03/_ato2007-2010/20099decreto/d6949.htm>.

Acesso em: 01 de Novembro de 2016 Cartilha de Acessibilidade na Web do W3C Brasil - Fascículo I - Introdução. Disponível em: http://www.w3c.br/pub/Materiais/PublicacoesW3C/cartilha-w3cbr-acessibilidade-we b-fasciculo-I.pdf.

Finizola, A. B., Raposo, E. H. S., Pereira, M. B. P. N., Gomes, W. S., de Araújo, A. L. S. O., \& Souza, F. V. C. (2014). O ensino de programação para dispositivos móveis utilizando o MIT-App Inventor com alunos do ensino médio. In Anais do Workshop de Informática na Escola (Vol. 20, No. 1, p. 337).

França, R. D., Silva, W. D., \& Amaral, H. D. (2012). Ensino de ciência da computação na educação básica: Experiências, desafios e possibilidades. In XX Workshop sobre Educação em Computação.

Gomes, T. C., \& de Melo, J. C. (2013). App inventor for android: Uma nova possibilidade para o ensino de lógica de programação. In Anais dos Workshops do Congresso Brasileiro de Informática na Educação (Vol. 2, No. 1).

MARATONA APP. (2014). Maratona de Aplicativos. Disponível em: <http://www.maratonadeaplicativos.com.br/>. Acesso em: 07/06/2018.

MIT - Massachusetts Institute of Technology. (2012) App Inventor for Android, <appinventor.mit.edu>, Acesso em: 07/06/2018.

Passerino, L. M., Santarosa, L. M. C., \& Tarouco, L. M. (2006). Pessoas com Autismo em Ambientes Digitais de Aprendizagem: estudo dos processos de Interação Social e Mediação. In Brazilian Symposium on Computers in Education (Simpósio Brasileiro de Informática na Educação-SBIE) (Vol. 1, No. 1, pp. 51-60).

Ribeiro, J. P., Manso, M. A., \& Borges, M. (2016). Dinâmicas com App Inventor no Apoio ao Aprendizado e no Ensino de Programação. In Anais do Workshop de Informática na Escola (Vol. 22, No. 1, p. 271).

Scaico, P. D., de Lima, A. A., Azevedo, S., da Silva, J. B. B., Azevedo, S., Paiva, L., Raposo, E. H., Alencar, Y.,\& Mendes, J. (2013). Ensino de programação no ensino médio: Uma abordagem orientada ao design com a linguagem scratch. Revista Brasileira de Informática na Educação, 21(02), 92. 